\title{
Predictive factors of the accelerated transepithelial corneal cross-linking outcomes in keratoconus
}

Mi Tian ${ }^{1,2,3+}$, Weijun Jian ${ }^{1,2,3 \dagger}$, Xiaoyu Zhang ${ }^{1,2,3}$, Ling Sun ${ }^{1,2,3}$, Yang Shen ${ }^{1,2,3}$ and Xingtao Zhou ${ }^{1,2,3^{*}}$

\begin{abstract}
Background: This study aimed to evaluate the clinical outcomes and assess preoperative characteristics that may predict outcomes in keratoconus 1 year after accelerated transepithelial corneal cross-linking (ATE-CXL).

Methods: This prospective study included 93 eyes of 84 consecutive keratoconus patients with 1-year follow-up after ATE-CXL. Preoperative characteristics included corneal astigmatism, anterior chamber depth, anterior chamber volume, radius of curvature, posterior elevation, central corneal thickness (CCT), thinnest corneal thickness, steepest meridian keratometry, flattest meridian keratometry, and the maximum keratometry (Kmax). Data were obtained preoperatively and at 1, 3, 6, and 12 months postoperatively. The patient eyes were grouped into 3 subgroups according to CCT and Kmax values to observe the changes of keratoconus progression.
\end{abstract}

Results: All patients were successfully operated without complications at any follow-up time point. Mean changes of Kmax from baseline at 6 and 12 months were $-0.60 \pm 2.21 \mathrm{D}(P=0.011)$ and $-0.36 \pm 1.58 \mathrm{D}(P=0.030)$, respectively. Eyes with a thinner CCT and higher Kmax values exhibited a tendency for topographic flattening of $\geq 1.0 \mathrm{D}(P=0.003$; $P=0.003)$. In the subgroup comparison, the Kmax values decreased significantly at 6 and 12 months after ATE-CXL in the group with $C C T \leq 450 \mu \mathrm{m}(P=0.018$ and $P=0.045)$; the Kmax values of the group with $\mathrm{Kmax}>65.0 \mathrm{D}$ decreased significantly at 6 months postoperatively $(P=0.025)$.

Conclusion: ATE-CXL is a safe and effective treatment for keratoconus patients. Patients with thinner CCT and higher Kmax values are more likely to benefit from ATE-CXL.

Keywords: Keratoconus, Accelerated transepithelial corneal cross-linking, Prospective study, Corneal thickness, Keratometry

\section{Background}

Keratoconus is a progressive degeneration disease, in which the cornea becomes thinner, leading to irregular astigmatism and irreversible loss of vision [1]. Corneal cross-linking $(\mathrm{CXL})$ is one of the treatments aimed at stabilizing the progression of keratoconus [2-4].

\footnotetext{
*Correspondence: doctzhouxingtao@163.com

${ }^{\dagger} \mathrm{Mi}$ Tian and Weijun Jian contributed equally to this work.

1 Eye Institute and Department of Ophthalmology, Eye and ENT Hospital,

Fudan University, No. 19 Baoqing Road, Shanghai 200031, China

Full list of author information is available at the end of the article
}

During the CXL procedure, a photochemical reaction is initiated to create extra or new chemical chains in the corneal stroma [5]. CXL enhances corneal hardness by increasing the covalent binding between collagen fibers [6-8]. Previously, studies have reported the efficacy of CXL in the visual and morphological improvement of keratoconus [9-14]. Accelerated transepithelial corneal cross-linking (ATE-CXL) is a treatment based on CXL, which can preserve the integrity of the cornea without removing the corneal epithelium and Bowman's layer (epithelium-on). It can also increase patients' cooperation by shortening the time of riboflavin infiltration original author(s) and the source, provide a link to the Creative Commons licence, and indicate if changes were made. The images or other third party material in this article are included in the article's Creative Commons licence, unless indicated otherwise in a credit line to the material. If material is not included in the article's Creative Commons licence and your intended use is not permitted by statutory regulation or exceeds the permitted use, you will need to obtain permission directly from the copyright holder. To view a copy of this licence, visit http://creativecommons.org/licenses/by/4.0/. The Creative Commons Public Domain Dedication waiver (http://creativeco mmons.org/publicdomain/zero/1.0/) applies to the data made available in this article, unless otherwise stated in a credit line to the data. 
and ultraviolet radiation. Our team has reported on the long-term safety and efficacy of ATE-CXL as a treatment for adolescent and adult keratoconus [15-18]. However, the efficacy of CXL may vary among patients. Therefore, more reports need to be published to help predict the postoperative results; this will help clinicians choose the treatment and gauge complications. Thus, the following question arises: Can preoperative factors be used to predict the possibility of stabilizing progression or improving corneal morphology after CXL?

Previous studies have reported how preoperative factors, including maximum keratometry (Kmax), central corneal thickness (CCT), thinnest corneal thickness (TCT) and the cone position affected the clinical outcomes of conventional corneal cross-linking (C-CXL; epithelium-off) [19-23] and accelerated corneal crosslinking (A-CXL; epithelium-off) [24, 25]. In the recent study [17], Zhang found that patients with thinner TCT showed more decreasing in average keratometry after ATE-CXL. However, the relationship between preoperative factors and postoperative outcomes of ATE-CXL are yet to be elucidated. Here, we evaluated the topographical outcomes and analyzed the preoperative parameters that may impact the corneal morphological changes of keratoconus patients up to 1 year after ATE-CXL.

\section{Patients and methods}

\section{Patients}

The collected data consisted of 93 eyes (44 right eyes and 49 left eyes) from 84 patients (60 male and 24 female, mean age: $24.51 \pm 4.99$ years) with progressive keratoconus diagnosed at the Eye and ENT Hospital of Fudan University in Shanghai, China. All recruited patients were treated by ATE-CXL (registration number: ChiCTROIC-16008181 29/03/2016). This study was approved by the Ethics Committee of the Eye and ENT Hospital of Fudan University and adhered to the tenets of the Declaration of Helsinki. Informed consent was obtained from all patients after a detailed explanation of the procedure prior to treatment.

The following features were defined as inclusion criteria for the study: 1) age over 18 years, 2) progressive keratoconus, defined as an increase in Kmax values, spherical refractive equivalent or astigmatism of $>1 \mathrm{D}$ in 1 year, 3) the TCT $>380 \mu \mathrm{m}, 4)$ no ophthalmological disease affecting the cornea. Exclusion criteria were as follows: 1) worn rigid gas permeable lenses and soft contact lenses of more than 4 and 2 weeks, respectively, 2) stromal scarring in the cornea, 3) allergy to any eye drops, 4) an ocular surgical history. The baseline characteristics are presented in Table 1.
Table 1 Demographics and characteristics of patients (Mean \pm SD)

\begin{tabular}{lll}
\hline & Mean \pm SD & Range \\
\hline Age (year) & $24.51 \pm 4.99$ & $(18,37)$ \\
Corneal astigmatism (D) & $3.81 \pm 2.58$ & $(0.1,12.2)$ \\
ACD $(\mathrm{mm})$ & $3.40 \pm 0.27$ & $(2.76,4.08)$ \\
ACV $(\mu \mathrm{L})$ & $205.09 \pm 27.66$ & $(133,269)$ \\
K1 $(D)$ & $48.65 \pm 5.44$ & $(39.2,65.1)$ \\
K2 $(D)$ & $52.47 \pm 6.52$ & $(39.6,71.6)$ \\
Kmax $(D)$ & $60.25 \pm 10.10$ & $(40,91.4)$ \\
CCT $(\mu m)$ & $473.75 \pm 41.34$ & $(386,589)$ \\
TCT $(\mu m)$ & $455.37 \pm 43.55$ & $(381,579)$ \\
\hline
\end{tabular}

$A C D$ anterior chamber depth, $A C V$ anterior chamber volume, $K 1$ steepest meridian keratometry, K2 flattest meridian keratometry, Kmax maximum keratometry, CCT central corneal thickness, TCT thinnest corneal thickness

\section{Grouping and result judgment criteria}

Patients were divided into three groups based on their $\mathrm{CCT}$ and the Kmax values preoperatively.

1) According to CCT: Group 1, CCT $>500 \mu \mathrm{m}$; group 2, CCT 450 to $500 \mu \mathrm{m}$; and, group $3, \mathrm{CCT}<450 \mu \mathrm{m}$.

2) According to Kmax: Group 1, Kmax values $>65$ D; group 2, Kmax values 55 to $65 \mathrm{D}$; and, group 3, Kmax values $<55 \mathrm{D}$.

Keratoconus progression was defined as an increase of $>1.0 \mathrm{D}$ in Kmax values, stabilization was defined as a change between $-1.0 \mathrm{D}$ and $+1.0 \mathrm{D}$, and regression was defined as a decrease of $>1.0 \mathrm{D}$.

\section{Measurements}

All patients underwent slitlamp biomicroscopy examination, endothelial cell density (ECD) and best corrected visual acuity (BCVA) assessments preoperatively and postoperatively. Anterior segment tomography parameters obtained by Pentacam (OCULUS Optikgeräte $\mathrm{GmbH}$; Wetzlar, Germany), including corneal astigmatism, anterior chamber depth (ACD), anterior chamber volume (ACV), CCT, TCT, steepest meridian keratometry (K1), flattest meridian keratometry (K2), Kmax, anterior radius of curvature (ARC), posterior radius of curvature (PRC), posterior central elevation (PCE), posterior mean elevation (PME), were recorded preoperatively and at $1,3,6$, and 12 months post operation. The ARC and PRC taken from a $3.0-\mathrm{mm}$ optical zone centered on the thinnest point. The PME was calculated as the mean of 27 points in the central $4.0 \mathrm{~mm}$ zone of the posterior corneal surface.

\section{Surgical procedure}

The procedure was performed under sterile conditions in the operating room of the Eye and ENT Hospital of 
Fudan University in Shanghai, China. ATE-CXL was started with topical anesthesia using oxybuprocaine hydrochloride eye drops. After placing a lid speculum, the ParaCel solution $(0.25 \%$ riboflavin and benzalkonium chloride, Avedro) was instilled for $4 \mathrm{~min}$ in the trephine (66 vision Tech, China) which was placed in central cornea. Then the cornea was soaked in the VibeX Xtra solution (0.25\% riboflavin solution, Avedro) for $6 \mathrm{~min}$. Subsequently, the cornea was rinsed with a balanced salt solution. Ultraviolet-A radiation with Avedro's KXL System (Avedro, Inc) was given at an irradiance of $45 \mathrm{~mW} / \mathrm{cm}^{2}$ in pulsed mode (1s on, 1s off) for $5 \mathrm{~min}$ and $20 \mathrm{~s}$, delivering a dose of $7.2 \mathrm{~J} / \mathrm{cm}^{2}$. During irradiation, a balanced salt solution was applied to prevent the cornea from dehydration. After the irradiation, a bandage contact lens was patched on the eye, which was removed after 3 days. Postoperatively, patients received topical antibiotics (levofloxacin) and artificial tears ( 4 times per day for 1 week and 1 month, respectively). Then, $0.1 \%$ fluorometholone was prescribed seven times per day; the dose frequency was tapered gradually over 2 weeks.

\section{Data analysis}

Statistical analysis was performed with SPSS. V23.0 (SPSS, Chicago, IL). Descriptive statistical data were displayed as mean \pm standard deviation (SD) for continuous data. The variables were investigated using the Kolmogorov-Smirnov test to determine their normality and homogeneity. Each follow-up result was compared with the preoperative values, and the results were analyzed by the paired t-test, Wilcoxon rank-sum test, and repeated measures analyses of variance with Bonferroni-adjusted post-hoc comparisons. A logistic regression analysis was done at the last follow-up visit to predict the presence or absence of regression (decrease in Kmax values of $\geq 1 \mathrm{D}$ ), based on sex, age, and preoperative corneal astigmatism, K1, K2, Kmax, CCT, TCT, ACD, ACV, and cone position. The intergroup comparison of subgroups divided by Kmax and CCT were analyzed by Chi-square test. A $P$-value of $<0.05$ was deemed statistically significant.

\section{Results}

All operations were successfully completed without postoperative complications. No other adverse event including endoethelial damage was observed in any eye. ECD at baseline was $3047.20 \pm 296.29$ cells $/ \mathrm{mm}^{2}$, and was $2996.48 \pm 265.47$ cells $/ \mathrm{mm}^{2}$ at 12 months postoperatively $(P=0.152)$. No significant changes were found in BCVA (in logMAR units), which was $0.32 \pm 0.15$ preoperatively and $0.28 \pm 0.13$ at 1 year post-ATE-CXL $(P=0.085)$.

\section{Corneal topography outcomes}

The Kmax values were $60.25 \pm 10.10 \mathrm{D}$ preoperatively, and $60.79 \pm 10.5 \mathrm{D}, 60.15 \pm 9.84 \mathrm{D}, 59.65 \pm 9.58 \mathrm{D}$, and $59.88 \pm 9.92 \mathrm{D}$ postoperatively at $1,3,6$, and 12 months, respectively. Mean changes of Kmax from baseline at $1,3,6$, and 12 months were $0.54 \pm 1.51 \mathrm{D}(P=0.001)$, $-0.09 \pm 1.46 \mathrm{D}(P=0.534),-0.60 \pm 2.21 \mathrm{D}(P=0.011)$ and $-0.36 \pm 1.58 \mathrm{D}(P=0.030)$, respectively. Pre- and postoperative Kmax, K1, and K2 values are shown in Fig. 1.

The TCT values were $455.37 \pm 43.55 \mu \mathrm{m}$ preoperatively, and $453.71 \pm 43.88 \mu \mathrm{m}, 454.74 \pm 46.76 \mu \mathrm{m}, 452.19 \pm 46.22 \mu \mathrm{m}$, and $451.22 \pm 46.51 \mu \mathrm{m}$ postoperatively at $1,3,6$, and 12 months, respectively. Mean changes of TCT from baseline at $1,3,6$, and 12 months were $-1.66 \pm 11.90 \mu \mathrm{m}(P=0.183)$, $-0.62 \pm 14.49 \mu \mathrm{m} \quad(P=0.679),-3.17 \pm 12.94 \mu \mathrm{m} \quad(P=0.020)$ and $-4.15 \pm 15.78 \mu \mathrm{m}(P=0.013)$, respectively. The CCT values showed a reduction in pachymetry at 1 month postoperatively. Pre- and postoperative TCT and CCT values are presented in Fig. 1.

The corneal astigmatism decreased from $3.81 \pm 2.58 \mathrm{D}$ preoperatively to $3.63 \pm 2.52 \mathrm{D}$ and $3.60 \pm 2.43 \mathrm{D}$ at 3 and 6 months postoperatively ( $P=0.049$ and $P=0.048$ ). Furthermore, the ACD values decreased from preoperatively to 12 months postoperatively ( $3.40 \pm 0.27$ vs $3.39 \pm 0.25, P=0.012$ ).

Compared with baseline, PCE significantly decreased from $6.76 \pm 0.74 \mu \mathrm{m}$ preoperatively to $6.73 \pm 0.74 \mu \mathrm{m}$ at 1 month postoperatively $(P=0.004)$, whereas not significantly different at 3, 6 and 12 months following ATE-CXL. No significant changes were observed in PRC, PCE and PME at any time point during follow-up $(P>0.05)$ (Table 2).

\section{Factors affecting the keratoconus progression}

To assess the factors affecting progression, logistic regression was used. It was found that the preoperative values of CCT and Kmax were predictors determining progression postoperatively $(P=0.003 ; P=0.003)$. However, there was no significant correlation between other preoperative parameters and the regression existence at 12 months (Table 3 ).

\section{Progress status after ATE-CXL}

In all patients, the progress status at 12 months postoperatively was as follows: progressive $(18.3 \%)$, stable (53.8\%), and regressive (27.9\%). Additionally, 14 (15.1\%) treated eyes showed progression with increases of Kmax by $>1-2$ D, $2(2.2 \%)$ eyes showed increases of Kmax by $>2-3 \mathrm{D}$, and $1(1.1 \%)$ treated eye showed an increase of Kmax by $3.2 \mathrm{D}$.

\section{Grouping according to CCT}

The stablization rates in group $1(\mathrm{CCT}>500 \mu \mathrm{m}), 2$ $(500 \mu \mathrm{m}>\mathrm{CCT} \geq 450 \mu \mathrm{m})$ and $3(\mathrm{CCT}<450 \mu \mathrm{m})$ were 


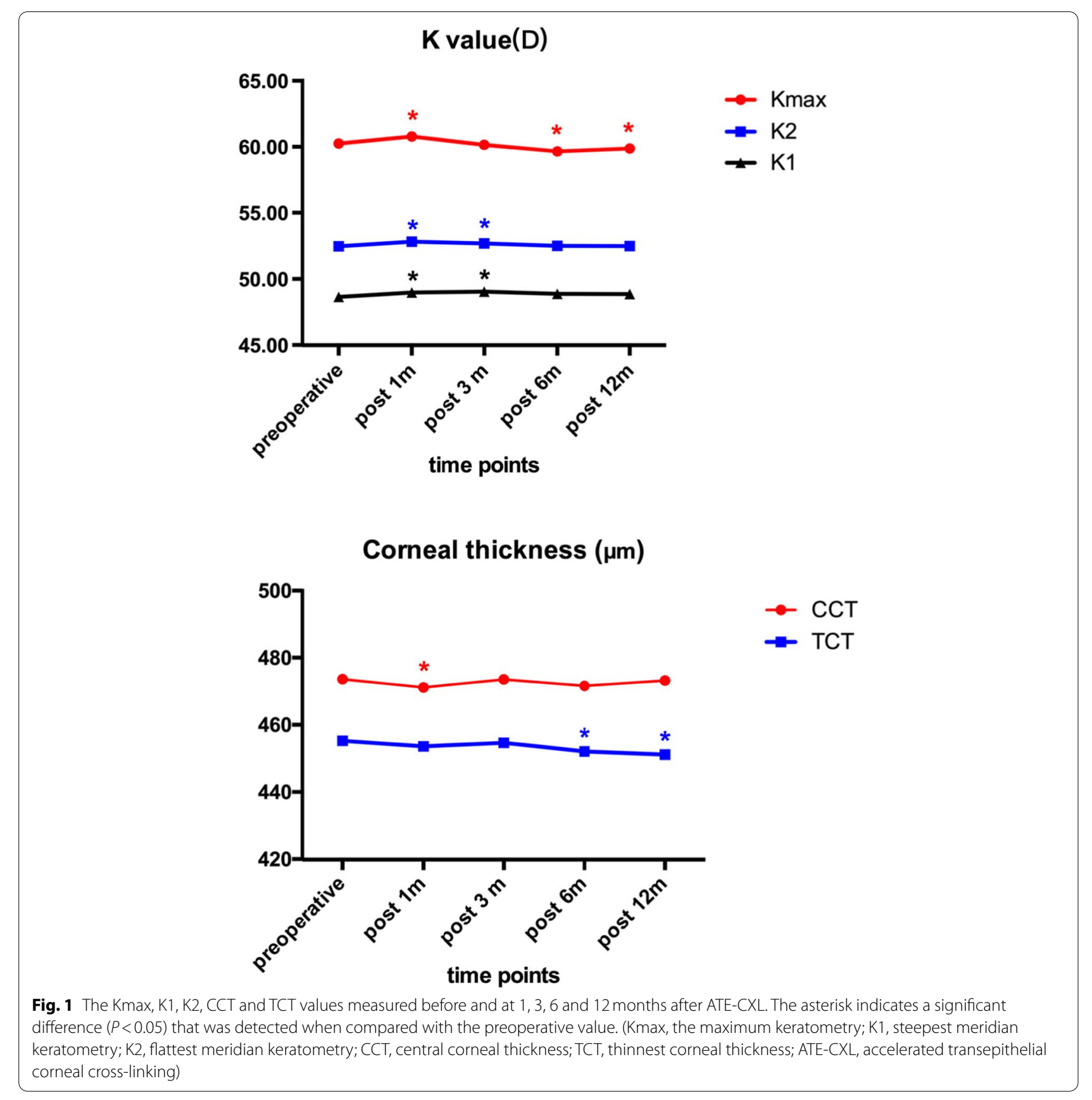

as follows: $96.3,78.0$ and $76.0 \%$; The regression rates in three groups were as follows: 0, 14.7 and $20 \%$. There were significant differences in status rates among three groups $(P=0.018)$. The progress status of each group is presented in Fig. 2.

The $\mathrm{K} 2$ values were increased at 1 month $(P=0.024)$ in group 1. In group 2, the ACV values were decreased at 3 months $(P=0.054)$; the Kmax values were increased at 1 month $(P=0.021)$; the TCT values decreased significantly from $454.85 \pm 20.42 \mu \mathrm{m}$ preoperatively to $449.68 \pm 22.45 \mu \mathrm{m}$ at 6 months postoperatively $(P=0.025)$. In group 3 , the ACD values were significantly decreased from preoperatively to 12 months postoperatively $(P=0.008)$; the Kmax values showed a significant reduction at 6 and 12 months postoperatively $(P=0.018$ and $P=0.045)$.

\section{Grouping according to Kmax}

The stablization rates in group 1 ( $K \max >65 \mathrm{D}), 2$ (65 $\mathrm{D}>\mathrm{Kmax} \geq 55 \mathrm{D})$ and $3(\mathrm{Kmax}<55 \mathrm{D})$ were as follows: 
Table 2 The ARC, PRC, PCE and PME values measured before and after ATE-CXL

\begin{tabular}{llllll}
\hline & Preoperative & Post 1 m & Post 3 m & Post 6 m & Post 12 m \\
\hline ARC $(\mathrm{mm})$ & $6.76 \pm 0.74$ & $6.73 \pm 0.74$ & $6.73 \pm 0.74$ & $6.75 \pm 0.74$ & $6.74 \pm 0.74$ \\
$P$ value & & $(P=0.004)$ & $(P=0.050)$ & $(P=0.619)$ & $(P=0.357)$ \\
PRC $(\mathrm{mm})$ & $5.26 \pm 0.70$ & $5.26 \pm 0.71$ & $5.25 \pm 0.71$ & $5.25 \pm 0.70$ & $5.26 \pm 0.71$ \\
$P$ value & & $(P=0.283)$ & $(P=0.237)$ & $(P=0.277)$ & $(P=0.744)$ \\
PCE $(\mu \mathrm{m})$ & $51.71 \pm 34.12$ & $52.57 \pm 35.43$ & $52.78 \pm 37.08$ & $53.32 \pm 36.35$ & $52.98 \pm 36.58$ \\
$P$ value & & $(P=0.469)$ & $(P=0.606)$ & $(P=0.310)$ & $(P=0.832)$ \\
PME $(\mu \mathrm{m})$ & $-18.25 \pm 12.63$ & $-18.32 \pm 12.60$ & $-18.23 \pm 12.59$ & $-18.15 \pm 12.37$ & $-18.33 \pm 12.61$ \\
$P$ value & & $(P=0.154)$ & $(P=0.716)$ & $(P=0.643)$ & $(P=0.797)$ \\
\hline
\end{tabular}

$A T E-C X L$ accelerated transepithelial corneal cross-linking, $A R C$ anterior radius of curvature, $P R C$ posterior radius of curvature, $P C E$ posterior central elevation, $P M E$ posterior mean elevation

Table 3 Logistic regression analysis between the regression existence at 12 months and preoperative parameters

\begin{tabular}{ll}
\hline Parameter & P value \\
\hline PREOP Gender & 0.677 \\
PREOP Age & 0.055 \\
PREOP K1 & 0.291 \\
PREOP K2 & 0.231 \\
PREOP Kmax & $0.003^{*}$ \\
PREOP Corneal astigmatism & 0.474 \\
PREOP TCT & 0.563 \\
PREOP CCT & $0.003^{*}$ \\
PREOP ACD & 0.373 \\
PREOP ACV & 0.964 \\
PREOP COne eccentricity & 0.983 \\
PREOP ARC & 0.307 \\
PREOP PRC & 0.179 \\
PREOP PCE & 0.414 \\
PREOP PME & 0.216 \\
\hline
\end{tabular}

Dependent factor: the regression existence at 12 months. PREOP preoperative; $\mathrm{K} 1$, steepest meridian keratometry, K2 flattest meridian keratometry, Kmax maximum keratometry, TCT thinnest corneal thickness, CCT central corneal thickness, $A C D$ anterior chamber depth, $A C V$ anterior chamber volume, $A R C$ anterior radius of curvature, $P R C$ posterior radius of curvature, $P C E$ posterior central elevation; $P M E$, posterior mean elevation.

${ }^{*} P<0.05$

26.9, 48.6 and $73.4 \%$; The regression rates in three groups were as follows: 50.0, 29.8 and 13.3\%. There were significant differences in status rates among three groups $(P=0.011)$. The progress status of each group is presented in Fig. 2.

In group 1 , the $A C D$ values showed significant decrease at 12 months $(P=0.039)$; the ACV values were significantly decreased at 3 and 6 months $(P=0.006$ and $P=0.020)$; the $K \max$ values were increased at 1 month $(73.03 \pm 6.91 \mathrm{D}$ vs $74.03 \pm 7.48 \mathrm{D}, P=0.026)$, but showed a significant decrease at 6 months postoperatively $(71.37 \pm 7.09 \mathrm{D}, P=0.025)$. In group 2 , the $\mathrm{ACV}$ values were decreased at 3 months $(P=0.054)$ postoperatively; no statistically significant $(P>0.05)$ changes were found for Kmax values during the 1-year follow-up. In group 3 , the $\mathrm{K} 2$ and $K \max$ values significantly increased from preoperatively to 1 month postoperatively $(P=0.001$, $P=0.047)$. The progress status of each group is presented in Fig. 2.

\section{Discussion}

CXL is an effective treatment for halting keratoconus progression. It makes the corneal collagen links compactly through a photochemical reaction, by the use of riboflavin and ultraviolet-A radiation [2-6]. ATE-CXL, however, is a newer treatment, and the relationship between the preoperative parameters and clinical outcome should be evaluated.

In this study, the Kmax decreased by $0.60 \pm 2.21 \mathrm{D}$ and $0.36 \pm 1.58 \mathrm{D}$ at 6 and 12 months postoperatively, suggesting that ATE-CXL is an effective treatment for keratoconus and can halt its progression. Toprak et al. [21] reported that Kmax significantly decreased from $54.54 \pm 5.50 \mathrm{D}$ preoperatively to $53.52 \pm 5.18 \mathrm{D}$ at the 1-year follow-up of 96 eyes after C-CXL; similar results were observed by Greenstein et al. [22] and Badawi et al. [26] Our results were also in concordance with these studies. According to the previous literature, the keratoconus eyes had a significantly higher ACD than normal eyes [27], and this value increased with the severity of keratoconus [28]. Our study found that ACD decreased at 12 months postoperatively, also suggesting that ATECXL is an effective treatment for halting the progression of keratoconus.

We found that corneal astigmatism decreased at 3 months and 6 months postoperatively, respectively. Badawi et al. [29] reported that corneal astigmatism decreased significantly in adolescent keratoconus after C-CXL during the 1-year follow-up. However, there was no significant difference in corneal astigmatism at 12 months postoperatively compared with the preoperative result in our study. The difference may be related to 


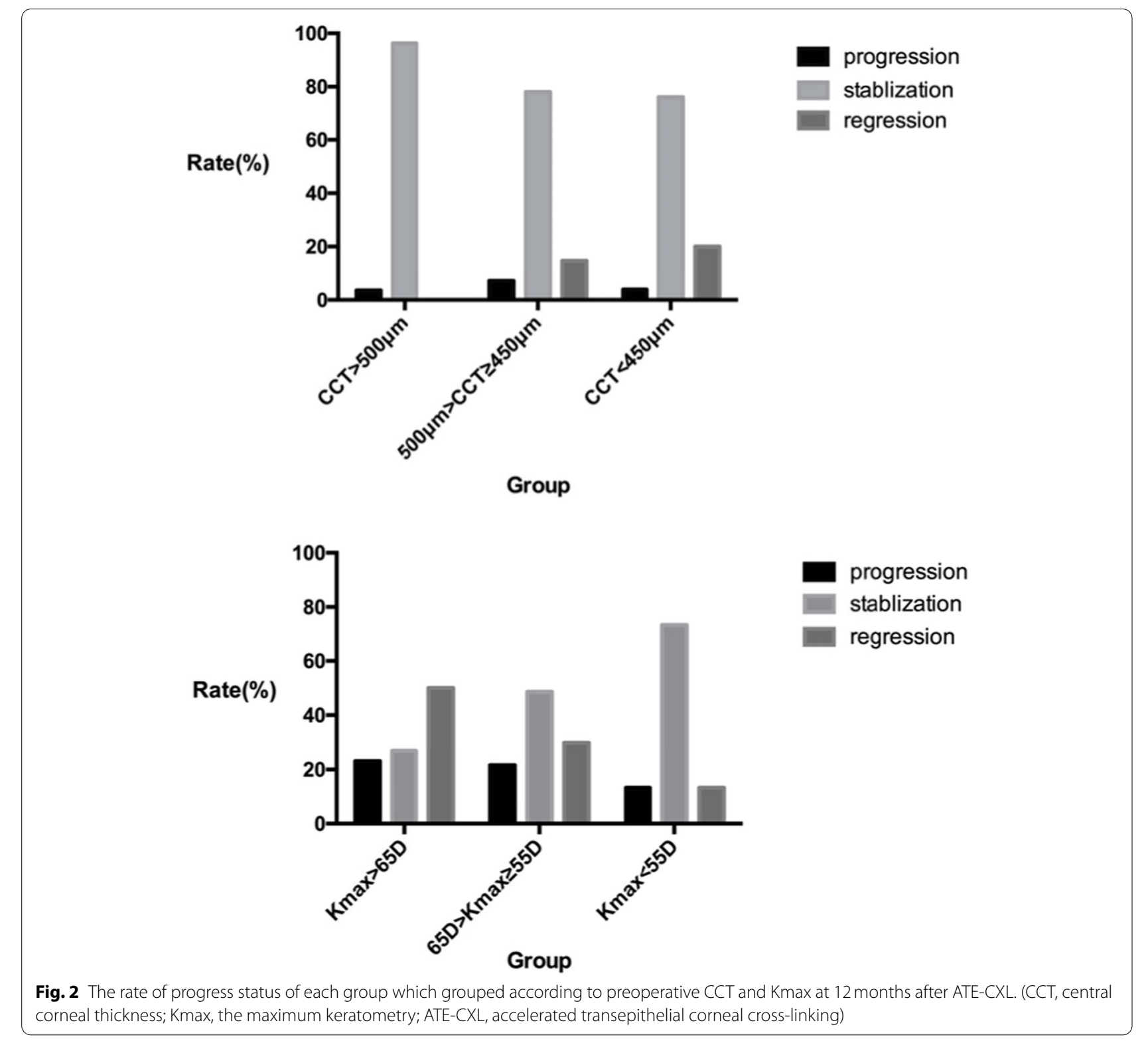

the age of the patient, as the corneal morphology of teenagers is easier to change. In addition, Hashemi et al. [30] found no significant difference in astigmatism at 4 years after C-CXL, suggesting that the alteration after C-CXL should be observed for a longer time. In this study, ARC, PRC, PCE and PME did not show significant changes at postoperative 12 months compared with the baseline, indicating that ATE-CXL may have prevented or delayed the progress of corneal ectasia and maintained structural stability of the cornea at 1 year after treatment.

Our results showed that CCT decreased at 1 month postoperatively, but there was no significant difference observed at 3,6, and 12 months follow-up. There was an initial sharp decrease in corneal thickness possibly due to a decrease in the interlamellar space and compaction of stroma. However, after a period of time, the corneal thickness increased again due to the remodeling of collagen fibers [31]. Our study also found that TCT decreased by $3.17 \pm 12.94 \mu \mathrm{m}$ and $4.15 \pm 15.78 \mu \mathrm{m}$ at 6 and 12 months postoperatively. A previous study reported that TCT decreased significantly from $460.11 \pm 47.15 \mu \mathrm{m}$ to $430.65 \pm 61.90 \mu \mathrm{m}$ during the 1-year follow-up after C-CXL [21]. Ozer et al. [24] found that TCT decreased significantly by $21.7 \pm 33.6 \mu \mathrm{m}$ at 1 year after C-CXL. Similar results were observed by Sarac et al. [23], where TCT decreased significantly from $430.2 \pm 54.4 \mu \mathrm{m}$ to 
$411.5 \pm 62.6 \mu \mathrm{m} 2$ years after C-CXL. Compared with the results of these studies, our results showed less decrease in TCT. The possible reason is that the epithelium is removed during C-CXL, while it is preserved in ATECXL; therefore, although TCT decreased significantly in this study, the decrease was lesser than that in previous studies of C-CXL.

In this study, we could not disregard $18.3 \%$ of treated eyes showed a tendency for progression at 1 year after ATE-CXL, which was a relatively big proportion comparing to those who were treated by C-CXL in some studies. The main reason might be the severe keratoconus accounted for a high proportion in this study (55.9\% patients with Kmax greater than 58 D), so that the probability of progression was relatively higher. Similarly, in Kuechler et al's study [32], the incidence of progression at 1 year after C-CXL was $23 \%$ in treating keratoconus eyes with Kmax values $\geq 58 \mathrm{D}$. However, compared with $\mathrm{C}$-CXL, the ATE-CXL is more safe, noninvasive and makes patient more comfortable.

Here, the preoperative parameters and the changes of Kmax were analyzed by logistic regression analysis. It was found that the thinner preoperative CCT or greater Kmax values have greater probability of flattening $\geq 1.0$ $\mathrm{D}$ in Kmax values after ATE-CXL, which indicates that ATE-CXL is an effective treatment for severe keratoconus. Ozer et al. [24] found that the change in Kmax after C-CXL was related to CCT in 4-year-follow-up. In contrast to our study, Toprak et al. [21] found that Kmax values alteration were related to age. Here, the age of the study population was $<30$ years ( $84.9 \%$ of all cases), that might have affected statistics. Godefrooij et al. [20] and Wisse et al. [19], reported that the change of Kmax values was related to the cone eccentricity after $\mathrm{C}-\mathrm{CXL}$, but here, the preoperative cone position of most patients was within $3 \mathrm{~mm}$ ( $82.8 \%$ of all cases), which could have affected the results.

In order to observe the effect after ATE-CXL under different preoperative conditions, we grouped the patients according to CCT and Kmax values before operation. We found that the probability of flattening (Kmax decrease of $\geq 1.0 \mathrm{D})$ gradually increased from 0 to $20 \%$ with the decrease of CCT thickness. The more serious keratoconus has thinner cornea, and an increase in infiltration depth of riboflavin leads to deeper effect in cornea during CXL. Therefore, the thicker cornea has the shallower depth of infiltration in stoma, and the effect of CXL may be affected. Toprak et al. [21] reported that patients with $\mathrm{TCT}<450 \mu \mathrm{m}$ suggested a decrease in Kmax after C-CXL, whereas Sarac et al. [23] reported an ease in progression after CXL, as thinner cornea represents more severe keratoconus, making the possibility of progress greater. The contradictory results suggest that the effects of CXL with thinner cornea should be confirmed by more cases. Zhang observed that the patients with TCT less than $450 \mu \mathrm{m}$ had the greater decreasing in average keratometry after ATE-CXL [17] in 42 eyes from 37 patients, which was similar to our results.

Our results also showed that the probability of flattening (Kmax reduction of $\geq 1.0 \mathrm{D}$ ) gradually decreased from 50 to $13.3 \%$ with the decrease of Kmax, suggesting that the patients with greater preoperative Kmax values have a better effect after ATE-CXL, which could be partly attributable to the relatively deep cross-linking in eyes with advanced keratoconus. However, our results also showed that the progressive rate increased with the rise of Kmax, and the reason might be the natural progression rate of keratoconic eyes in advanced subgroup are more likely higher than in mild subgroup. The study by Koc et al. [25] showed similar results in the 1-year follow-up after C-CXL. Greenstein et al. [22] reported that the patients with a Kmax value of $\geq 55.0 \mathrm{D}$, were more likely to improve by decreasing more than $2.0 \mathrm{D}$ in Kmax. According to Koller et al. [33], patients with a Kmax value of $\geq 54.0 \mathrm{D}$ had a greater decrease in Kmax. These studies suggested that the preoperative Kmax values had an impact on the Kmax values after CXL; however, the specific relationship needs to be further explored.

There are some limitations in our study. The follow-up time was only 1 year, which can be increased to obtain the long-term clinical effects of the treatment. In addition, there are some published studies that have assessed the corneal biomechanical properties of patients with keratoconus by Corvis-ST, which can be added in the follow-up examination to evaluate the safety of ATE-CXL.

Our study suggests that ATE-CXL is a safe and effective treatment for patients with keratoconus. Patients with thinner CCT and higher Kmax values were most likely to improve after ATE-CXL, but the long-term effects are yet to be elucidated and need further observation.

\section{Acknowledgements}

Not applicable.

\section{Authors' contributions}

Literature screening and selection was performed by MT and WJ. XZhou participated in the design of the study. WJ and MT drafted the manuscript. MT revised the manuscript. XZhang carried out the statistical analysis. LS and YS prepare and review of the manuscript. XZhou has given final approval of the version to be published. All authors read and approved the final manuscript.

\section{Funding}

This work was supported by grants from the National Natural Science Foundation of China (Grant No. 81770955); Joint research project of new frontier technology in municipal hospitals (SHDC12018103); Project of Shanghai Science and Technology (Grant No.17411950200).

Availability of data and materials

The datasets used and analyzed during the current study are available from the corresponding author on reasonable request. 


\section{Declarations}

\section{Ethics approval and consent to participate}

This study was approved by the Ethics Committee of the Eye and ENT Hospital of Fudan University and was carried out following the tenets of the Declaration of Helsinki.

\section{Consent for publication}

Not applicable.

\section{Competing interests}

There are no conflicts of interest to declare for all authors.

\section{Author details}

'Eye Institute and Department of Ophthalmology, Eye and ENT Hospital, Fudan University, No. 19 Baoqing Road, Shanghai 200031, China. ${ }^{2}$ NHC Key Laboratory of Myopia (Fudan University); Key Laboratory of Myopia, Chinese Academy of Medical Sciences, Shanghai, China. ${ }^{3}$ Shanghai Research Center of Ophthalmology and Optometry, Shanghai, China.

Received: 18 February 2021 Accepted: 24 December 2021

Published online: 03 January 2022

\section{References}

1. Rabinowitz YS. Keratoconus. Surv Ophthalmol. 1998;42(4):297-319. https://doi.org/10.1016/s0039-6257(97)00119-7 [published Online First: 1998/03/11].

2. Vinciguerra P, Camesasca Fl, Albe E, et al. Corneal collagen cross-linking for ectasia after excimer laser refractive surgery: 1-year results. J Refract Surg. 2010;26(7):486-97. https://doi.org/10.3928/1081597X-20090910-02 [published Online First: 2009/09/24].

3. Wollensak G, Spoerl E, Seiler T. Riboflavin/ultraviolet-a-induced collagen crosslinking for the treatment of keratoconus. Am J Ophthalmol. 2003;135(5):620-7. https://doi.org/10.1016/s0002-9394(02)02220-1 [published Online First: 2003/04/30].

4. Vinciguerra R, Romano MR, Camesasca Fl, et al. Corneal cross-linking as a treatment for keratoconus: four-year morphologic and clinical outcomes with respect to patient age. Ophthalmology. 2013;120(5):908-16. https://doi.org/10.1016/j.ophtha.2012.10.023 [published Online First: 2013/01/08]

5. Kohlhaas M, Spoerl E, Schilde T, et al. Biomechanical evidence of the distribution of cross-links in corneas treated with riboflavin and ultraviolet a light. J Cataract Refract Surg. 2006;32(2):279-83. https://doi.org/10.1016/j. jcrs.2005.12.092 [published Online First: 2006/03/28].

6. Beshtawi IM, O'Donnell C, Radhakrishnan H. Biomechanical properties of corneal tissue after ultraviolet-A-riboflavin crosslinking. J Cataract Refract Surg. 2013;39(3):451-62. https://doi.org/10.1016/j.jcrs.2013.01.026 [published Online First: 2013/03/20].

7. Spoerl E, Huhle M, Seiler T. Induction of cross-links in corneal tissue. Exp Eye Res. 1998;66(1):97-103. https://doi.org/10.1006/exer.1997.0410 [published Online First: 1998/06/17].

8. Spoerl E, Wollensak G, Seiler T. Increased resistance of crosslinked cornea against enzymatic digestion. Curr Eye Res. 2004;29(1):35-40. https://doi. org/10.1080/02713680490513182 [published Online First: 2004/09/17].

9. Wollensak G. Crosslinking treatment of progressive keratoconus: new hope. Curr Opin Ophthalmol. 2006;17(4):356-60. https://doi.org/10.1097/ 01.icu.0000233954.86723.25 [published Online First: 2006/08/11].

10. Caporossi A, Baiocchi S, Mazzotta C, et al. Parasurgical therapy for keratoconus by riboflavin-ultraviolet type a rays induced cross-linking of corneal collagen: preliminary refractive results in an Italian study. J Cataract Refract Surg. 2006;32(5):837-45. https://doi.org/10.1016/j.jcrs.2006.01.091 [published Online First: 2006/06/13].

11. Vinciguerra P, Albe E, Trazza S, et al. Refractive, topographic, tomographic, and aberrometric analysis of keratoconic eyes undergoing corneal crosslinking. Ophthalmology. 2009;1 16(3):369-78. https://doi.org/10.1016/j. ophtha.2008.09.048 [published Online First: 2009/01/27].

12. O'Brart DP, Chan E, Samaras K, et al. A randomised, prospective study to investigate the efficacy of riboflavin/ultraviolet a $(370 \mathrm{~nm})$ corneal collagen cross-linkage to halt the progression of keratoconus. Br J Ophthalmol. 2011;95(11):1519-24. https://doi.org/10.1136/bjo.2010.196493 [published Online First: 2011/02/26].

13. Wittig-Silva C, Chan E, Islam FM, et al. A randomized, controlled trial of corneal collagen cross-linking in progressive keratoconus: three-year results. Ophthalmology. 2014;121(4):812-21. https://doi.org/10.1016/j. ophtha.2013.10.028 [published Online First: 2014/01/08]

14. Hersh PS, Greenstein SA, Fry KL. Corneal collagen crosslinking for keratoconus and corneal ectasia: one-year results. J Cataract Refract Surg. 2011;37(1):149-60. https://doi.org/10.1016/j.jcrs.2010.07.030 [published Online First: 2010/12/25].

15. Tian M, Jian W, Sun L, et al. One-year follow-up of accelerated transepithelial corneal collagen cross-linking for progressive pediatric keratoconus. BMC Ophthalmol. 2018;18(1):75. https://doi.org/10.1186/s12886018-0739-9 [published Online First: 2018/03/11]

16. Shen $Y$, Jian $W$, Sun $L$, et al. One-year follow-up of changes in corneal densitometry after accelerated $(45 \mathrm{~mW} / \mathrm{cm} 2)$ Transepithelial corneal collagen cross-linking for keratoconus: a retrospective study. Cornea. 2016;35(11):1434-40. https://doi.org/10.1097//CO.0000000000000934 [published Online First: 2016/10/18]

17. Zhang $X$, Sun L, Chen Y, et al. One-year outcomes of Pachymetry and epithelium thicknesses after accelerated (45 mW/cm(2)) Transepithelial corneal collagen cross-linking for keratoconus patients. Sci Rep. 2016;6:32692. https://doi.org/10.1038/srep32692 [published Online First: 2016/09/07]

18. Tian M, Jian W, Zhang X, et al. Three-year follow-up of accelerated transepithelial corneal cross-linking for progressive paediatric keratoconus. $\mathrm{Br}$ J Ophthalmol. 2020. https://doi.org/10.1136/bjophthalmol-2019-315260 [published Online First: 2020/02/14].

19. Wisse RP, Godefrooij DA, Soeters N, et al. A multivariate analysis and statistical model for predicting visual acuity and keratometry one year after cross-linking for keratoconus. Am J Ophthalmol. 2014;157(3):519-25 e1-2. https://doi.org/10.1016/j.ajo.2013.11.001 [published Online First: 2013/11/12].

20. Godefrooij DA, Boom K, Soeters N, et al. Predictors for treatment outcomes after corneal crosslinking for keratoconus: a validation study. Int Ophthalmol. 2017;37(2):341-8. https://doi.org/10.1007/s10792-016-0262z [published Online First: 2016/05/26].

21. Toprak I, Yaylali V, Yildirim C. Factors affecting outcomes of corneal collagen crosslinking treatment. Eye (Lond). 2014;28(1):41-6. https://doi.org/ 10.1038/eye.2013.224 [published Online First: 2013/10/19].

22. Greenstein SA, Hersh PS. Characteristics influencing outcomes of corneal collagen crosslinking for keratoconus and ectasia: implications for patient selection. J Cataract Refract Surg. 2013;39(8):1133-40. https://doi.org/10. 1016/j.jcrs.2013.06.007 [published Online First: 2013/07/31]

23. Sarac O, Caglayan M, Cakmak HB, et al. Factors influencing progression of keratoconus 2 years after corneal collagen cross-linking in pediatric patients. Cornea. 2016;35(12):1503-7. https://doi.org/10.1097//CO.00000 00000001051 [published Online First: 2016/10/16].

24. Ozer MD, Batur M, Mesen S, et al. Long-term results of accelerated corneal cross-linking in adolescent patients with keratoconus. Cornea. 2019;38(8):992-7. https://doi.org/10.1097//CO.0000000000001975 [published Online First: 2019/04/30].

25. Koc M, Uzel MM, Tekin K, et al. Effect of preoperative factors on visual acuity, corneal flattening, and corneal haze after accelerated corneal crosslinking. J Cataract Refract Surg. 2016;42(10):1483-9. https://doi.org/ 10.1016/j.jcrs.2016.08.017 [published Online First: 2016/11/15].

26. Badawi AE, Abou Samra WA, El Ghafar AA. Predictive factors of the standard cross-linking outcomes in adult keratoconus: one-year follow-up. J Ophthalmol. 2017:2017:4109208. https://doi.org/10.1155/2017/4109208 [published Online First: 2017/09/28]

27. Edmonds CR, Wung SF, Pemberton B, et al. Comparison of anterior chamber depth of normal and keratoconus eyes using Scheimpflug photography. Eye Contact Lens. 2009;35(3):120-2. https://doi.org/10.1097/ ICL.0b013e31819cf5a6 [published Online First: 2009/05/08].

28. Hashemi H, Asharlous A, Aghazadeh Amiri M, et al. Intrasubject repeatability and Interdevice agreement of anterior chamber depth measurements by Orbscan and Pentacam in different grades of keratoconus. Eye Contact Lens. 2019;45(1):51-4. https://doi.org/10.1097/ICL.0000000000 000515 [published Online First: 2018/06/27] 
29. Badawi AE. Accelerated corneal collagen cross-linking in pediatric keratoconus: one year study. Saudi J Ophthalmol. 2017;31(1):11-8. https://doi. org/10.1016/j.sjopt.2017.01.002 [published Online First: 2017/03/25].

30. Hashemi H, Seyedian MA, Miraftab M, et al. Corneal collagen cross-linking with riboflavin and ultraviolet a irradiation for keratoconus: long-term results. Ophthalmology. 2013;120(8):1515-20. https://doi.org/10.1016/j. ophtha.2013.01.012 [published Online First: 2013/04/16]

31. Padmanabhan P, Rachapalle Reddi S, Rajagopal R, et al. Corneal collagen cross-linking for keratoconus in pediatric patients-long-term results. Cornea. 2017;36(2):138-43. https://doi.org/10.1097//CO.0000000000001102 [published Online First: 2017/01/07].

32. Kuechler SJ, Tappeiner C, Epstein D, et al. Keratoconus progression after corneal cross-linking in eyes with preoperative maximum Keratometry values of 58 diopters and steeper. Cornea. 2018;37(11):1444-8. https:// doi.org/10.1097//CO.0000000000001736 [published Online First: 2018/08/30]

33. Koller T, Pajic B, Vinciguerra $\mathrm{P}$, et al. Flattening of the cornea after collagen crosslinking for keratoconus. J Cataract Refract Surg. 2011;37(8):1488-92. https://doi.org/10.1016/j.jcrs.2011.03.041 [published Online First: 2011/07/26].

\section{Publisher's Note}

Springer Nature remains neutral with regard to jurisdictional claims in published maps and institutional affiliations.

- fast, convenient online submission

- thorough peer review by experienced researchers in your field

- rapid publication on acceptance

- support for research data, including large and complex data types

- gold Open Access which fosters wider collaboration and increased citations

- maximum visibility for your research: over $100 \mathrm{M}$ website views per year

At BMC, research is always in progress.

Learn more biomedcentral.com/submissions 